\title{
Incompatibility of Metric Structure in Recombination Space
}

\author{
Tazid Ali \\ Department of Mathematics, Dibrugarh University, \\ Dibrugarh, Assam, India
}

\author{
Chandra Kanta Phukan \\ Department of Mathematics, Dibrugarh University, \\ Dibrugarh, Assam, India
}

\begin{abstract}
Recombination is a binary operator in the set $X$ of all chromosomes, i.e., it is a function $R: X \times X \rightarrow P(X)$, the power set of $X$. This notion of recombination induces a closure operator given by $\quad C l(A)=\{R(x, y):(x, y)$ $\in A \times A\}$. However the neighbourhood structure so induced is not in general a topological space. In this paper we have attempted to study the recombination space in fuzzy setting by assigning possibilities to each offspring under the recombination operator. We have shown that a fuzzy pretopology is naturally generated in the recombination set. We have studied two unequal crossover models viz. unrestricted and restricted in this setting. We have further observed that both the models are incompatible with metric measures.
\end{abstract}

Keywords: Fuzzy Recombination Set, Fuzzy Metric, Fuzzy Closure, Fuzzy Metrizable, Unequal Crossover,

2010, Mathematical Subject Classification: Primary 92C40, 92D15, 92D20; Secondary 54A05, 54A40, 54E35,

\section{INTRODUCTION}

All living organisms consist of cells. In each cell there is the same set of chromosomes. Chromosomes are strings of DNA and serves as a model for the whole organism. A chromosome consists of genes, blocks of DNA. Each gene encodes a particular protein. Basically it can be said, that each gene encodes a trait, for example colour of eyes. Possible settings for a trait (e.g. blue, brown) are called alleles. Each gene has its own position in the chromosome. This position is called locus. Complete set of genetic material (all chromosomes) is called genome. Particular set of genes in genome is called genotype. The genotype with later development is the base for the organism's phenotype, its physical and mental characteristics, such as eye colour, intelligence etc. Natural selection acts on genetic variation that comes from two principal sources: mutation and recombination. In recombination DNA molecules interact with one another to bring about a rearrangement of the genetic information or content in an organism. In eukaryotic system, the process that is responsible for chromosomal crossing-over during meiosis, which leads to offspring's having different combinations of genes from those of their parents. The frequency of recombination is actually not the same for all gene combinations. One may say that recombination is greatly influenced by the proximity of one gene to another. If two genes are located close together on a chromosome, the likelihood that a recombination event will separate these two genes is less than if they were farther apart. Genetic linkage describes the tendency of genes to be inherited together as a result of their location on the same chromosome

Mathematical recombination is based on the notion of recombination function $R: X \times X \rightarrow P(X)$. The recombination set consist of all the possible offspring that are obtained by recombining two parents chromosomes say $x$ and $y$ using a given family of cross-over operators. This can be formalized by a weaker topological structure, known as neighbourhood space, which satisfies basic axioms of it under the closure operator [5]. A new way of constructing recombination spaces is introduced and the topological features of the resulting hypergraphs are analyzed in [8]. It is shown that types, which are neighbours in the point mutation space, are also neighbours in the recombination space, i.e. mutation and recombination spaces are homomorphic. This implies that the shapes of the fitness functions explored by mutation and recombination are similar. However, the potential of one-point and two-point recombination operators to explore the fitness landscape may differ dramatically from uniform recombination operators or mutation operators because of the limited number of recombinant types they can produce. In the classical model of the recombination sets are considered as a sub-basis of the neighbourhood filters and construct the coarsest pretopology in which the recombination set are neighbourhoods and by adding the intersections of any finite number of recombination sets to the basis[6]. The structure of recombination space arising from few unequal cross-over is modeled in the context of pretopological space. On the other hand different possibilities of the recombinants (offspring) suggest a fuzzy version on the set of all chromosomes. In this paper we have outlined a general method to study the fuzzy topological features induced by recombination processes, where each offspring under fuzzy recombination operator posses some possible values. The main advantage of a fuzzy topological approach proposed here is that the tools can be applied to more and more general cases of recombination mechanism. We have discussed some theorems and definitions arising in fuzzy recombination set. We have shown that a fuzzy pretopology naturally arises in the recombination set of two crossover models viz. unrestricted unequal crossover and restricted unequal crossover and observed that both the models are incompatible with a fuzzy metric measure.

\section{RECOMBINATION SETS}

The abstract definition of recombination spaces is based on the notion of the recombination functions $R: X \times X \rightarrow P(X)$. Given a pair of parental chromosomes $x$ and $y$ the recombination set $R(x, y)$ consists of all chromosomes that can be obtained by recombining $x$ and $y$ using a given family of crossover operators. Consider the following properties: 
(X1) $\{x, y\} \subseteq R(x, y)$,

(X2) $R(x, y)=R(y, x)$,

(X3) $R(x, x)=\{x\}$,

(X4) For all $z \in R(x, y)$ holds $|R(x, z)| \leq|R(x, y)|$

The first condition is only for notational convenience. The second condition is a simple symmetry requirement. The third says that no new types are created from a single type by recombination and the fourth condition defines the topological implications of recombination. It essentially says that recombinants are more "similar" to the parental types than the two parental types. This is the idea that recombinants are mixtures of the two parental types. Furthermore this condition says that the more "similar" two types are, the fewer types can be created by recombination.

A generalized recombination structure satisfies (X1) and (X2). The proper recombination structures of homologous crossover satisfy also (X3) and (X4) [8]. Recombination functions are considered as transit functions or $P$ - structures if it satisfies (X1), (X2), (X3) [7]. It seems natural to interpret $R(x, y)$ as neighbourhoods of $x$ for each $y \in X$. By (X1) we have $x \in R(x, y)$ for all $x, y$. Thus the recombination sets form a neighbourhood basis if and only if for all $x, y, z$ there is a $v$ such that

$$
R(x, v) \subseteq R(x, y) \cap R(x, z)
$$

In general this condition will not be satisfied. We may, however, consider the recombination sets as a sub-basis of the neighbourhood filters and construct the coarsest pretopology in which the recombination sets are neighbourhoods by adding the intersections of any finite number of recombination sets to the basis. In the case of finite genome sets $X$ there is always a smallest neighbourhood $N(x)$, i.e., a minimal element of the neighbourhood basis. This is true in general if the neighbourhood filters have a finite basis, i.e., in Alexandroff spaces. Provided $X$ is finite we can extract the vicinities directly from the (sub) basis of recombination sets:

$$
N(x)=\bigcap_{x \in X} R(x, y)
$$

If $X$ is infinite, however, the vicinity $N(x)$ defined in the Equ. (2) need not be a neighbourhood of $x$ in general. The intersection of a finite number of neighbourhoods of course is again a neighbourhood. Equ.(2) however defines neighbourhoods if the size of the recombination sets $R(x, y)$ is bounded. The closure operator associated with a recombination function was introduced in $[3,6]$ as

$$
C l(A)=\{R(x, y):(x, y) \in A \times A\}
$$

The closure operator in (3) defines a neighbourhood space, which is not sub-additive in general.

\section{FUZZY SET AND FUZZY TOPOLOGY}

In this section we discuss some preliminaries on fuzzy sets[2] and fuzzy topology[3] that will be required in the sequel. Further we propose some new notions.

Fuzzy (sub)sets are generalization of classical (sub)sets. In a classical subset an element of the universal set either belongs to the subset or not which can be identified by the corresponding characteristic function $\chi_{A}: X \rightarrow\{0,1\}$ such that $\chi_{A}(x)=1$, if $x \in A$ and $\chi_{A}(x)=0$, if $x \notin A$. Here the boundary of a fuzzy subset is not precisely defined and so an element of the universal set belongs to the fuzzy subset with some level of membership which can be identified by the membership function $\mu_{\mathrm{A}}$. In short fuzzy set expresses the concept of graded membership.

Mathematically a fuzzy set $A$ of a universal set $X$ is a function

$$
\mu_{A}: X \rightarrow[0,1]
$$

For each $x \in X, \mu_{A}(x)$ is called the membership grade of $x$ in $A$. For convenience the fuzzy subset as well as the corresponding membership function is represented by $\mu$.

Given two fuzzy sets $A$ and $B$, their standard intersection $A \cap B$, standard union $A \cup B$ and standard complement $A^{\mathrm{c}}$ are defined for all $x \in X$ by the equations

$$
\begin{aligned}
& \mu_{A \cap B}(x)=\min \left[\mu_{A}(x), \mu_{B}(x)\right] \\
& \mu_{A} \cap B(x)=\max \left[\mu_{A}(x), \mu_{B}(x)\right] \\
& \mu_{A}{ }^{\mathrm{c}}(x)=1-\mu(x)
\end{aligned}
$$

For infinite collection of fuzzy subsets, $\min$ and $\max$ are respectively replaced by infimum and supremum.

A fuzzy set $A$ is said to be contained in a fuzzy set $B$, denoted by $A \subseteq B$, if

$$
\mu_{A}(x) \leq \mu_{B}(x) \quad \forall x \in X
$$

Definition 3.1 [1] A fuzzy pretopology on a set $X$ is described by an application $C l$ of $I^{X}$ into $I^{X}$, which verifies:

P $1: C l(\phi)=\phi$,

P2 : $C l(A) \supset A$ for every $A \in I^{X}$,

$(X, C l)$ is then said to be a fuzzy pretopological space. Sometimes we suppose that $\mathrm{Cl}$ verifies some additional properties, for instance:

Definition 3.2 [1] Let ( $X, C l)$ be a fuzzy pretopological space, and let us consider the following properties:

P 3 : For every $A$ and $B \in I^{X}$, such that $\mathrm{A} \supset \mathrm{B}$

we have $C l(A) \supset C l(B) .(X, C l)$ is then said to be of type I.

P $4:$ For every $A$ and $B \in I^{X}$

we have $C l(A \cup B)=C l(A) \cup C l(B) .(X, C l)$ is then said to be of type D.

P 5 : For every $A \in I^{X}$ we have 
$C l^{2}(A)=C l(C l(A))=C l(A) .(X, C l)$ is said to be of type $\mathrm{S}$.

A fuzzy pretopological space which is of type $\mathrm{I}, \mathrm{D}, \mathrm{S}$ is a fuzzy topological space and $\mathrm{Cl}$ is its Kuratowsky closure.

Definition 3.3 [1] A family of fuzzy preneighbourhoods at the point $x \in X$ is a family $\mathbf{B}(x)$ of fuzzy subsets $\mu_{V}$ which verify $\mu_{V}(x)=1$

Definition 3.4 [1] Let $(X, C l)$ be a pretopological space. A family of fuzzy sets $\mathbf{B}(x)$ of $X$ is called preneighbourhood basis of $(X, C l)$ if it satisfies

(i) $\mu(x)=1 \quad \forall \mu \in \mathbf{B}(x)$,

(ii) For $\mu, v \in \mathbf{B}(x) \exists \gamma \in \mathbf{B}(x): \gamma \subseteq \mu \wedge \nu$.

Definition 3.5 [1] Let $\varphi$ be an application of $I^{X}$ in to $[0,1], \varphi$ is said to be a degree of non-vacuity ( it associates to every fuzzy subset a number which represents the fact that it is more or less void ) if it verifies:

(i) $\varphi(\phi)=0$,

(ii) $\varphi(A)=1$ if there exists $x$ such that $\mu_{A}(x)=1$,

(iii ) $A \supset B$ implies $\varphi(A) \supset \varphi(B)$.

In particular $\varphi(A)=\sup _{x \in X} \mu_{A}(x)$ is the degree of non-vacuity. If we define a fuzzy subset $\bar{\mu}$ of $X$ such that

$$
\bar{\mu}(x)=\inf _{v \in \mathbf{B}(x)} \varphi(v \wedge \mu)
$$

Then we get a fuzzy closure operator on $X$. This gives a connection between preneighbourhoods and closure operator.

Definition 3.6 [3] A fuzzy point on a set $X$ is a fuzzy subset of $X$ that takes non-zero value at exactly one point of $X$. If the fuzzy point takes non-zero value $r \in[0,1]$ at the point $x \in X$, then it is denoted by $x_{r}$.

Definition 3.7 A element $x \in X$ is said to belong to a fuzzy subset $\mu$ of $X$ (i.e., $x \in \mu$ ) if $\mu(x)>0$.

There are different definitions of fuzzy metric [4] in the literature. We propose a new one in this paper.

Definition 3.8 A fuzzy metric $\tilde{d}$ on $X$ is a classical metric on $\tilde{X}$ ( the set of all fuzzy point of $X$ ), satisfying the extra condition $\tilde{d}\left(x_{r}, y_{s}\right)=\tilde{d}\left(x_{s}, y_{r}\right)$.

Example 3.1 If $d$ be a metric defined on a set $X$, then a function $\tilde{d}: \tilde{X} \times \tilde{X} \rightarrow[0, \propto)$ defined as

$$
\tilde{d}\left(x_{r}, y_{s}\right)=d(x, y)+|r-s|
$$

is a fuzzy metric on $X$.

(i) $\tilde{d}\left(x_{r}, y_{s}\right) \geq 0, \quad \forall x_{r}, y_{s} \in \tilde{X}$, Since

$$
d(x, y) \geq 0 \text { and }|r-s| \geq 0 \forall x, y \in X .
$$

(ii ) $\tilde{d}\left(x_{r}, y_{s}\right)=0$

$$
\Leftrightarrow d(x, y)+|s-r|=0
$$

$$
\begin{aligned}
& \Leftrightarrow d(x, y)=0 \text { and }|r-s|=0 \\
& \Leftrightarrow x=y \& r=s \Leftrightarrow x_{r}=y_{s},
\end{aligned}
$$

(iii) $\tilde{d}\left(x_{r}, y_{s}\right)=\tilde{d}\left(y_{s}, x_{r}\right) \quad \forall x_{r}, y_{s} \in \tilde{X}$

Since, $\tilde{d}\left(x_{r}, y_{s}\right)=d(x, y)+|r-s|$

$$
\begin{aligned}
& =d(y, x)+|s-r| \\
& =\tilde{d}\left(y_{s}, x_{r}\right)
\end{aligned}
$$

(iv ) Let us consider $x_{r}, y_{s}, z_{t}$ are three fuzzy points on $X$, then

$$
\begin{aligned}
\tilde{d}\left(x_{r}, z_{t}\right) & +\tilde{d}\left(z_{t}, y_{s}\right) \\
& =d(x, z)+|r-t|+d(z, y)+|t-s| \\
& \geq d(x, \mathrm{z})+d(z, y)+|r-t+t-s| \\
& \geq d(x, y)+|r-s| \\
& =\tilde{d}\left(x_{r}, y_{s}\right) \\
\tilde{d}\left(x_{r}, z_{t}\right) & +\tilde{d}\left(z_{t}, y_{s}\right) \geq \tilde{d}\left(x_{r}, y_{s}\right)
\end{aligned}
$$

Hence, $\tilde{d}$ this is a fuzzy metric on $X$.

Definition 3.9 Let $\tilde{d}$ is a fuzzy metric on $X$, let for $x \in X$ and $\varepsilon>0$

$$
\begin{aligned}
& B\left(x_{r}, \varepsilon\right)=\left\{y_{s}: \tilde{d}\left(x_{r}, y_{s}\right)<\varepsilon\right\} \\
& B^{\prime}\left(x_{r}, \varepsilon\right)=\left\{y_{s}: \tilde{d}\left(x_{r}, y_{s}\right) \leq \varepsilon\right\}
\end{aligned}
$$

are called open and closed ball with centred at $x \&$ radius $\varepsilon$ respectively. These two balls can be looked upon as fuzzy subsets of $X$ with the membership functions given by

$$
\begin{gathered}
B\left(x_{r}, \varepsilon\right)(y)=\sup \left\{s: \tilde{d}\left(x_{r}, y_{s}\right)<\varepsilon\right\} \text { and } \\
B^{\prime}\left(x_{r}, \varepsilon\right)(y)=\sup \left\{s: \tilde{d}\left(x_{r}, y_{s}\right) \leq \varepsilon\right\} .
\end{gathered}
$$

If $\tilde{d}$ is a fuzzy metric on $X$, then the fuzzy topology induced by $\tilde{d}$ is define by the neighbourhood basis

$\mathbf{B}(x)=\{B(x, \varepsilon): \varepsilon>0\}$ for each $x \in X$.

Definition 3.10 A fuzzy pretopological space is said to be weakly metrizable if there is a fuzzy metric

$\tilde{d}: \tilde{X} \times \tilde{X} \rightarrow[0, \propto)$ and set $A, A^{\prime} \subset(0, \propto)$ such that $\mathbf{B}(x)=\{B(x, \varepsilon): \varepsilon \in A\} \cup\left\{B^{\prime}(x, \varepsilon): \varepsilon \in A^{\prime}\right\}$, is a fuzzy preneighbourhoods basis of $X$, where $B(x, \varepsilon)$ and $B^{\prime}(x, \varepsilon)$ are open and closed ball respectively.

Definition 3.11 A fuzzy topological space is called $T_{\mathrm{o}}$ - space if for all $x \neq y \quad \exists \vee \in \mathbf{B}(y)$ such that $x \notin v$ i.e., $\vee(x)$ $=0$ or $\exists \gamma \in \mathbf{B}(x)$ such that $y \notin \gamma$ i.e., $\gamma(y)=0$.

Definition 3.12 A fuzzy topological space is called $T_{1}$ - space if for all $x \neq y \exists \vee \in \mathbf{B}(y)$ such that $x \notin v$ i.e., $v(x)=0$. It is clear from the definition that $T_{1}$ implies $T_{\mathrm{o}}$.

Definition 3.13 A fuzzy topological space is called $R_{\mathrm{o}}$, if for $x \in\{\bar{y}\}$ implies $y \in\{\overline{x\}}$ i.e., 
$\{\overline{y\}}(x)>0$ implies $\{\bar{x}\}(y)>0$ for all $x, y \in X$.

[ Here, the fuzzy sub set $\{x\}$ takes the value 1 only at the point $x$ and 0 at all other points ]

Theorem 3.1 A weakly metrizable fuzzy pretopological space $X$ is $R_{\mathrm{o}}$.

Proof : Since $X$ is weakly metrizable pretopological space $\exists \quad A, A^{\prime} \subset(0, \propto)$ such that

$\mathbf{B}(x)=\{B(x, \varepsilon): \varepsilon \in A\} \cup\left\{B^{\prime}(x, \varepsilon): \varepsilon \in A^{\prime}\right\}$,

Let $x \in\{\overline{y\}} \Rightarrow\{\bar{y}\}(x)>0$

$\Rightarrow$ inf $v \in \mathbf{B}(x) \varphi\left(y_{1} \wedge v\right)>0$

$\Rightarrow$ inf $v \in \mathbf{B}(x) \varphi\left(y_{1} \wedge v\right)>k^{\prime}$,

where $0<k^{\prime}<k=\inf v \in \mathbf{B}(x) \varphi\left(y_{1} \wedge v\right)$

$\Rightarrow \varphi\left(y_{1} \wedge v\right)>k^{\prime} \quad \forall v \in \mathbf{B}(x)$

$\Rightarrow \sup _{u \in X}\left(y_{1} \wedge v\right)(u)>k^{\prime} \quad \forall v \in \mathbf{B}(x)$

$\Rightarrow \sup _{u \in X}\left(y_{1}(u) \wedge \vee(u)>k^{\prime} \quad \forall v \in \mathbf{B}(x)\right.$

$\Rightarrow y_{1}(y) \wedge \vee(y)>k^{\prime} \quad \forall \vee \in \mathbf{B}(x)$

[ if $u \neq y, y_{1}(u)=0$ so $u=y$ ],

$\Rightarrow \boldsymbol{V}(y)>k^{\prime} \quad \forall \boldsymbol{V} \in \mathbf{B}(x), \quad$ since $y_{1}(u)=1$

$\Rightarrow B(x, \varepsilon)(y)>k^{\prime} \forall \varepsilon \in A$ or

$$
B^{\prime}(x, \varepsilon)(y)>k^{\prime} \quad \forall \varepsilon \in A^{\prime}
$$

Therefore for each $\varepsilon \in A$ or $\varepsilon \in A^{\prime} \exists$ at least one

$s_{\lambda}>k^{\prime}: y_{s_{\lambda}} \in B(x, \varepsilon)$ or $y_{s_{\lambda}} \in B^{\prime}(x, \varepsilon)$ such that

$\tilde{d}\left(x, y_{s_{\lambda}}\right)<\varepsilon \forall \varepsilon \in A$ or $\tilde{d}\left(x, y_{s_{\lambda}}\right) \leq \varepsilon \forall \varepsilon \in A^{\prime}$

$\Rightarrow \tilde{d}\left(y, x_{s_{\lambda}}\right)<\varepsilon \quad \forall \varepsilon \in A$ or

$\tilde{d}\left(y, x_{s_{\lambda}}\right)<\varepsilon \quad \forall \varepsilon \in A^{\prime}$ [ From Def. 3.6 ]

$\Rightarrow B(y, \varepsilon)(x) \geq s_{\lambda} \forall \varepsilon \in A$ or

$B^{\prime}(y, \varepsilon)(x) \geq s_{\lambda} \forall \quad \varepsilon \in A^{\prime}$

$\Rightarrow B(y, \varepsilon)(x)>k^{\prime} \forall \varepsilon \in A$ or

$B^{\prime}(y, \varepsilon)(x)>k^{\prime} \quad \forall \varepsilon \in A^{\prime}\left[\right.$ since $s_{\lambda}>k^{\prime}$ ]

$\Rightarrow \mu(x)>k^{\prime} \quad \forall \mu \in \mathbf{B}(y)$

$\Rightarrow x_{1}(x) \wedge \mu(x)>k^{\prime} \quad \forall \mu \in \mathbf{B}(y)$

$\Rightarrow \sup _{u \in X}\left\{x_{1}(u) \wedge \mu(u)\right\}>k^{\prime} \quad \forall \mu \in \mathbf{B}(y)$

$\Rightarrow \sup _{u \in \mathrm{X}}\left(x_{1} \wedge \mu\right)(u)>k^{\prime} \quad \forall \mu \in \mathbf{B}(y)$

$\Rightarrow \varphi\left(x_{1} \wedge \mu\right)>k^{\prime} \quad \forall \mu \in \mathbf{B}(y)$

$\Rightarrow \inf \varphi\left(x_{1} \wedge \mu\right) \geq k^{\prime} \quad \forall \mu \in \mathbf{B}(y)$

$\Rightarrow \inf \varphi\left(x_{1} \wedge \mu\right)>0 \quad \forall \mu \in \mathbf{B}(y)$, [since $0<k^{\prime}$ ]

$\Rightarrow\{\bar{x}\}(y)>0 \Rightarrow y \in\{\bar{x}\}$

Hence the fuzzy pretopology is $R_{\mathrm{o}}$.

Theorem 3.2 If $X$ be a fuzzy pretopological space then,

(i ) $X$ is $T_{1}$ if and only if $\{\overline{x\}}=\{x\}$ for all $x \in X$,

(ii) $T_{1}$ implies $R_{0}$.

Proof: ( i )

Let $y \in\{\overline{x\}} \Rightarrow\{\bar{x}\}(y)>0$

$\Rightarrow \inf \varphi\left(x_{1} \wedge v\right)>0 \quad \forall v \in \mathbf{B}(y)$

$\Rightarrow \varphi\left(x_{1} \wedge v\right)>0 \quad \forall v \in \mathbf{B}(y)$

$\Rightarrow \sup _{u \in X}\left\{\left(x_{1} \wedge v\right)(u)\right\}>0 \quad \forall v \in \mathbf{B}(y)$

$\Rightarrow \sup _{u \in X}\left\{x_{1}(u) \wedge \vee(u)\right\}>0 \quad \forall v \in \mathbf{B}(y)$

$\Rightarrow x_{1}(x) \wedge v(x)>0 \quad \forall v \in \mathbf{B}(y)$

[ if $u \neq x, x_{1}(u)=0$, so $u=x$ ]

$\Rightarrow \boldsymbol{V}(x)>0 \quad \forall \boldsymbol{V} \in \mathbf{B}(y)$

which is a contradiction, since, $X$ is $T_{1}, \quad \exists \gamma \in \mathbf{B}(y)$ such that $x \notin \gamma$ i.e., $\gamma(x)=0$. Consequently $\{\overline{x\}}=\{x\}$.

Conversely,

Let $\{\overline{x\}}=\{x\} \quad \forall x \in X$,

For $x \neq y$ we have $\{x\}(y)=0$

$\Rightarrow\{\bar{x}\}(y)=0$

$\Rightarrow \inf \varphi\left(x_{1} \wedge v\right)=0 \quad \forall v \in \mathbf{B}(y)$

$\Rightarrow \inf \left[\sup _{u \in X}\left\{\left(x_{1} \wedge v\right)(u)\right\}\right]=0 \quad \forall v \in \mathbf{B}(y)$

$\Rightarrow \inf \left[\sup _{u \in X}\left\{\left(x_{1}(u) \wedge \vee(u)\right\}\right]=0 \forall \vee \in \mathbf{B}(y)\right.$

$\Rightarrow \inf [\boldsymbol{V}(x)]=0 \quad \forall \boldsymbol{V} \in \mathbf{B}(y)$

$\Rightarrow v(x)=0 \quad \forall v \in \mathbf{B}(y)$

therefore $\exists v \in \mathbf{B}(y)$ such that $x \notin v$. Hence it is $T_{1}$.

(ii) Since, $X$ is $T_{1}$

$\Rightarrow\{\overline{x\}}=\{x\} \quad \forall x \in X$.

Now, $y \in\{\overline{x\}} \Rightarrow y \in\{x\} \Rightarrow\{x\}(y)>0$

$\Rightarrow x=y \Rightarrow x \in\{\bar{y}\}$. Hence $X$ is $R_{\mathrm{o}}$.

\section{FUZZY PRETOPOLOGY IN RECOMBINATION SPACE}

In general the entire recombinants yield from recombination event has different possibilities. If we assign some values of possibilities to each recombinant then the recombination set can be looked upon as a fuzzy set. We denote the fuzzy recombination set obtained from two chromosomes $x$ and $y$ by 
$\mu_{x y}$. The set $\mu_{x y}$ consists of all possible recombinants between chromosomes with $x$ and $y$ numbers of gene copies with different grade of membership. If $X$ denotes the set of chromosomes then the recombinant set corresponding to a pair $(x, y)$ is a membership function

$$
\mu_{x y}: X \rightarrow[0,1]
$$

Here, the fuzzy subset $\mu_{x y}$ can be considered as a fuzzy preneighbourhood of $x$ as well as $y$ for each $x, y \in X$ by considering $\mu_{x y}(x)=1$ and $\mu_{x y}(y)=1$ respectively. The concept of fuzzy pretopology on the set of chromosomes can be obtained from the closure operator defined by

$$
C l(\mu)(x)=\inf _{v \in \mathbf{B}(x)} \varphi(\nu \wedge \mu)
$$

where, $\mu$ is an arbitrary fuzzy subset on $X$ and $\mathbf{B}(x)$ is the set of all fuzzy preneighbourhood $x$. This closure function satisfies following conditions

$$
\begin{aligned}
& \text { (i) } C l(\phi)=\phi \\
& \text { (ii) } C l(\mu) \supseteq \mu \\
& \text { (iii ) If } \mu \supseteq \gamma \text { then } C l(\mu) \supseteq C l(\gamma) \text {. }
\end{aligned}
$$

Hence the fuzzy recombination set and the fuzzy closure defined in (4) satisfies the basic axioms of the type-I fuzzy pretopology, so we have a fuzzy pretopology on the set of all chromosomes.

\subsection{Unrestricted Unequal Crossover}

In the unrestricted unequal crossover model [6] an extreme form of unequal cross over is assumed that a crossover may happen with equal probability at all possible intergenic regions as well as at both ends of the gene cluster. Each possible crossover event produces two recombinant chromosomes. In most of the cases the recombination events will yield chromosomes with different number of gene copies than the original ones. The recombination set $R(x, y)$ consists of all possible recombinants between chromosomes with $x$ and $y$ copies of gene i.e., $R(x, y)=\{0,1, \ldots$, $x+y\}$, where $N(x)=R(x, 0)=\{0,1, \ldots, x\}$ represents the smallest neighbourhood. In the fuzzy pretopological model we can consider each element of $R(x, y)$ having different possibilities of occurrence..

Let us now consider $x \neq y$ and $x<y$ be two unequal chromosomes, then support of all preneighbourhoods of $y$ contains the elements of $R(y, 0)$ with different possibilities, which also contains $x$. There does not exist any such preneighbourhood of $y$ which does not contain $x$. So the space is not $T_{1}$. Theorem 3.2 implies that it is not $R_{0}$. Finally we can conclude form the Theorem 3.1 that the unequal unrestricted crossover space is not weakly fuzzy metrizable space. Hence this space is incompatible with fuzzy metric.

\subsection{Restricted Unequal Crossover}

In restricted unequal crossover model the number of genecopies changes by at most one compared to the parental chromosome numbers. The recombination sets for this model are

$$
\begin{aligned}
R(x, y)= & \{x-1, x, x+1\} \cup\{y-1, y, y+1\} \\
& \text { if } x, y>1 \\
R(x, 0)= & \{x-1, x, 0, x+1\} \quad \text { if } x>1 \\
R(0,0)= & \{0\}
\end{aligned}
$$

In this case $x$ and $x-1$ are two distinct chromosomes. The support of all preneighbourhoods of $x$ contains all the element of $R(x, 0)=\{x-1, x, 0, x-1\}$, which contains $x-1$. Therefore there does not exist any such preneighbourhood of $x$ which does not contain $x-1$. In a particular case support of all the preneighbourhoods of 1 contains all the element of $R(1,0)=\{0,1\}$ which contains 0 . Therefore no such neighbourhood of 1 exist which does not contains 0 . So, the space is not $T_{1}$. Once the space is not $T_{1}$, it is not $R_{0}$. Which gives the fuzzy pretopological space is not weakly metrizable and incompatible with fuzzy metric.

\section{CONCLUSION}

The main conclusion of our discussion is that a fuzzy pretopological notion is naturally generated in recombination space. We have seen that a fuzzy pretopology is uniquely determined by fuzzy closure operator defined on the set of all fuzzy subset of all chromosomes. Each fuzzy recombination set is treated as a fuzzy preneighbourhood of each of its points which determines the recombination set. The concept of nonvacuity is introduced to construct fuzzy closure of all fuzzy subsets on the recombination space. Weakly metrizable fuzzy pretopological space is defined to study the compatibility of metric in the fuzzy recombination set specially for the two crossover models namely unrestricted unequal crossover and restricted unequal crossover with few separation axioms and proofs in fuzzy environment, which eventually shows the models are incompatible with fuzzy metric distance defined on it.

6. ACKNOWLEDGEMENT: The first author is thankful to the Department of Science and Technology, Govt. of India for providing financial support under a research project.

\section{REFERENCES}

[1] Badard, R. 1981: Fuzzy pretopological spaces and their representation, Journal of mathematical analysis and applications, 81: 378-390.

[2] Klir, G. J., Yuan, B. 2003: Fuzzy Sets and Fuzzy Logic: Theory and Application, Prentice Hall of India Private Limited.

[3] Palaniappan, N. 2005: Fuzzy Topology, Narosa Publication House, India.

[4] Sharma, S. 2002: On Fuzzy Metric Space, Southeast Asian Bulletin of Mathematics, 26 : 133-145.

[5] Stadler, B.M.R., Stadler, P. F. 2002: Generalized topological spaces in evolutionary theory and combinational chemistry, J. Chem. Inf. Comput. Sci., 42: 577-585. 
[6] Stadler, B.M.R., Stadler, P. F., Shapk, M., Wanger, G. P. 2002: Recombination spaces, metrics, and pretopologies, Z. Phys. Chem., 216: 217-234.

[7] Stadler, B.M.R., Stadler, P.F. 2004: The Topology of Evolutionary Biology, I G. Ciobanu and G. Rozenerg, editors, Modelling in Molecular Biology, pages 267-286, Springer Verlag.
[8] Gitchoff, P., Wagner, G. P 1996: Recombination induced hypergraphs: A new approach to mutation-recombination isomorphism, Complexity, 2: 37-43. 